\title{
Asia-Pacific venous thromboembolism consensus in knee and hip arthroplasty and hip fracture surgery: Part 3. Pharmacological venous thromboembolism prophylaxis
}

\author{
Satit Thiengwittayaporn ${ }^{1}$, Nicolaas Budhiparama ${ }^{2}$, Chotetawan Tanavalee ${ }^{3}$, Saran Tantavisut ${ }^{4}$, Rami M. Sorial ${ }^{5}$, \\ Cao $\mathrm{Li}^{6}$, Kang- $\| \mathrm{Kim}^{7,8^{*}}$ (D) and The Asia-Pacific (AP) Region Venous Thromboembolism (VTE) Consensus Group
}

\section{Statements of Group 3: Pharmacological Venous Thromboembolism Prophylaxis}

1. Which pharmacological agents are widely accepted for venous thromboembolism (VTE) prophylaxis in knee and hip arthroplasty and hip fracture surgery?

Recommendation

The widely accepted pharmacological agents for VTE prophylaxis include aspirin, unfractionated heparin (UFH), low-molecular-weight heparin (LMWH), adjusted-dose vitamin $\mathrm{K}$ antagonists (VKAs), synthetic pentasaccharide factor Xa inhibitor (fondaparinux), oral factor Xa inhibitor, and direct thrombin inhibitor (DTI) anticoagulants.

Delegate vote: Agree 100\%, Disagree 0\%, Abstain 0\% (Unanimous Consensus)

\section{Justification}

The American College of Chest Physicians (ACCP) guidelines recommend the use of LMWH, low-dose UFH, VKA, fondaparinux, apixaban, dabigatran, rivaroxaban, or aspirin (all Grade 1B) for patients who undergo total knee arthroplasty (TKA) or total hip arthroplasty (THA) [1]. For patients who undergo hip fracture surgery, the guidelines recommend the use of $\mathrm{LMWH}$, low-

\footnotetext{
* Correspondence: drkim@khu.ac.kr

${ }^{7}$ Department of Orthopaedic Surgery, Center for Joint Diseases, Kyung Hee University Hospital at Gangdong, 892 Dongnam-ro, Gangdong-gu, Seoul 134-727, South Korea

${ }^{8}$ Department of Orthopaedic Surgery, School of Medicine, Kyung Hee University, Seoul, South Korea

Full list of author information is available at the end of the article
}

dose UFH, VKA, fondaparinux, or aspirin (all Grade 1B) [1]. Although many current guidelines have different recommendations, the variations rely on the risk of VTE, bleeding risk, and patient safety [2]. Overall, the ACCP recommends LMWH as an optimal pharmacological agent for VTE prophylaxis in patients undergoing THA, TKA, or hip fracture surgery [1].

The American Academy of Orthopaedic Surgeons (AAOS) guideline could not make absolute recommendations concerning the most effective prophylaxis agents [3]. As a result, the guideline provides orthopedic surgeons with flexibility regarding the use of different prophylactic regimens. When choosing the medicine, orthopedic surgeons should justify VTEs risk, bleeding risk, and patient safety.

Aspirin is safe because it provides a low risk of bleeding with no requirement for routine blood testing [2]. Several studies supported the use of aspirin for preventing VTE [2, 4]. A meta-analysis of using aspirin as a thromboprophylactic agent in knee and hip arthroplasty found that the overall rate of deep vein thrombosis (DVT) and pulmonary embolism (PE) in both groups after using aspirin was $1.2 \%$ and $0.6 \%$, respectively, and the rate of major bleeding was $0.3 \%$ [2]. The pooled mortality rate was only $0.2 \%$ [2]. A recent systematic review, including 11 relevant studies with various dosing regimens, concluded that aspirin could reduce VTE with a low risk of bleeding complication [4].

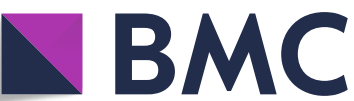

Part of Springer Nature
(9) The Author(s). 2021 Open Access This article is licensed under a Creative Commons Attribution 4.0 International License, which permits use, sharing, adaptation, distribution and reproduction in any medium or format, as long as you give appropriate credit to the original author(s) and the source, provide a link to the Creative Commons licence, and indicate if changes were made. The images or other third party material in this article are included in the article's Creative Commons licence, unless indicated otherwise in a credit line to the material. If material is not included in the article's Creative Commons licence and your intended use is not permitted by statutory regulation or exceeds the permitted use, you will need to obtain permission directly from the copyright holder. To view a copy of this licence, visit http://creativecommons.org/licenses/by/4.0/ The Creative Commons Public Domain Dedication waiver (http://creativecommons.org/publicdomain/zero/1.0/) applies to the data made available in this article, unless otherwise stated in a credit line to the data. 
LMWH was used instead of warfarin and UFH in clinical practice and VTE studies in knee and hip arthroplasty and hip fracture surgery with a particular efficacy [5]. It can be administered once daily without laboratory monitoring or dose adjustment [6]. Therefore, the use of LMWH is more convenient than UFH, and it has the advantage of less heparin-induced thrombocytopenia [6].

A systematic review and meta-analysis in 2019 suggests that fondaparinux is significantly superior to LMWH in reducing VTE for perioperative arthroplasty surgery [7]. However, the authors of that review advise that clinicians be aware of the higher risk of major bleeding, especially surgical site bleeding, with fondaparinux [7]. A metaanalysis of four randomized controlled trials (RCTs) showed that fondaparinux significantly reduced VTE incidence compared with enoxaparin in major orthopedic surgery [8]. The effect was consistent across all types of surgeries and all subgroups [8]. Major bleeding occurred more significantly frequently in the fondaparinux group [8]. However, the incidence of clinically relevant bleeding (leading to death or reoperation) did not differ between groups [8]. Another RCT showed that the fondaparinux group had a significantly lower incidence of VTE than the enoxaparin group in patients with TKA [9]. Major bleeding occurred more significantly frequently in the fondaparinux group, but there were no significant differences between the two groups in the incidence of bleeding leading to death or reoperation or occurring in a critical organ [9].

Direct factor Xa inhibitors work by binding to the active site of factor Xa, blocking the interaction with its substrate [1]. Examples of oral direct factor Xa inhibitors are rivaroxaban, apixaban, edoxaban, and betrixaban [1]. The ACCP recommends rivaroxaban and apixaban in the same manner as fondaparinux [1]. Rivaroxaban is a Food and Drug Administration (FDA)-approved oral direct factor Xa inhibitor that requires no monitoring [1]. There are numerous studies on rivaroxaban's effectiveness in preventing DVT [10-13]. In a comparison of extended rivaroxaban with short-term enoxaparin in THA, the results showed that DVT, nonfatal $\mathrm{PE}$, and all-cause mortality up to days 30-42 occurred at $2.0 \%$ in the rivaroxaban group, compared with $9.3 \%$ in the enoxaparin group [11]. When comparing rivaroxaban with enoxaparin in TKA, the results showed that DVT, nonfatal $\mathrm{PE}$, and all-cause mortality up to day 17 occurred at $6.9 \%$ in the rivaroxaban group, compared with $10.1 \%$ in the enoxaparin group, with a significant difference [11]. An RCT comparing apixaban with enoxaparin in TKA reported lower rates of clinically relevant bleeding with apixaban [14]. Two RCTs using apixaban showed its superior efficacy compared to enoxaparin $[15,16]$. Overall, there were no significant differences in the rates of major bleeding between apixaban and enoxaparin $[15,16]$.

The direct thrombin inhibitor dabigatran works by binding specifically to the active center of thrombin and inactivating free and fibrin-bound thrombin [17]. This process is reversible, leaving a small amount of free and active thrombin to control hemostasis [17]. In the USA, dabigatran etexilate is an FDA-approved oral direct thrombin inhibitor for the prevention of atrial fibrillation and stroke, but not for VTE prophylaxis after TKA and THA [17]. A study of dabigatran indicated that over 1215 days of treatment, dabigatran etexilate was not as effective as enoxaparin in preventing total VTE and mortality in patients undergoing TKA [17]. However, an RCT study demonstrated that, over 6-10 days of treatment, dabigatran etexilate was noninferior to enoxaparin for the prevention of VTE in patients undergoing TKA [18]. There was no significant difference in the frequency of major bleeding or the overall rate of adverse events between either dose of dabigatran etexilate and enoxaparin [18]. Similarly, another trial in patients undergoing THA with extended VTE prophylaxis reported the same outcomes [19]. Another trial demonstrated that extended prophylaxis with oral dabigatran etexilate was as effective as subcutaneous enoxaparin in reducing the risk of VTE after THA, and superior to enoxaparin in reducing the risk of major VTE, with similar safety profiles [20].

Warfarin was the first oral anticoagulant widely used in the USA and has been in use since 1954 [4]. It is a VKA that inhibits the synthesis of active vitamin-K-dependent coagulation factors [4]. Therapeutic anticoagulation is reached $24-72 \mathrm{~h}$ after the initial dose [21]. Usually, 5 or $10 \mathrm{mg}$ of warfarin is given the night before or the night of surgery, and then dosing is adjusted to maintain an international normalized ratio (INR) of 2.0 [21]. The rate of DVT using low-dose warfarin ranges from 35 to $59 \%$ [21]. When comparing warfarin to LMWH, LMWH was more effective in preventing DVT formation but showed no difference to warfarin in preventing symptomatic events, including PE [22, 23]. The risks of warfarin include bleeding and multiple drug interactions [21]. The use of warfarin also requires regular monitoring of the INR [21]. Mismetti et al. [24] conducted a meta-analysis of VKAs. They reported that VKAs were more significantly effective than a placebo or no treatment in reducing DVT and clinical PE [24]. However, there was a significantly higher rate of wound hematoma compared to the placebo [24]. By contrast, VKAs were significantly less effective than LMWH in preventing total DVT and proximal DVT [24]. The differences between VKAs and LMWH in major hemorrhage and wound hematoma were not significant [24].

\section{Is the protocol for pharmacological VTE prophylaxis similar among knee arthroplasty, hip arthroplasty, and hip fracture surgery? Recommendation}

No. Although a general protocol for pharmacological VTE prophylaxis can apply to all patients who undergo 
knee or hip arthroplasty or hip fracture surgery, different prophylaxis protocols should be considered based on the risk stratification of patients.

Delegate vote: Agree 97.2\%, Disagree 1.4\%, Abstain 1.4\% (Strong Consensus)

\section{Justification}

Recently, the AAOS and the ACCP developed new evidence-based guidelines for venous thromboembolic prophylaxis after total joint arthroplasty (TJA) [1, 3]. Based on a review of the available literature, the AAOS guideline panel was unable to recommend a specific prophylaxis regimen or duration of prophylaxis following routine TJA [3]. The optimal duration of thromboprophylaxis after TKA remains controversial. It is a common practice to administer prophylaxis using LMWH or UFH until discharge from hospital, usually 7-14 days after surgery [25]. International guidelines recommend extending thromboprophylaxis for up to 35 days following major orthopedic surgery, but the recommendation is weak because of moderate-quality evidence [26]. Extended (4-week) prophylaxis with fondaparinux can produce a $96 \%$ reduction in risk of DVT and an $89 \%$ reduction in risk of symptomatic VTE events relative to perioperative (1-week) prophylaxis [26]. As the only anticoagulant approved in the USA for thromboprophylaxis in patients with hip fractures, fondaparinux offers more effective prophylaxis against VTE without compromising safety [27].

According to the ACCP, patients with hip fractures are at high risk for DVT and PE [1]. In this patient group, the ACCP recommends using LMWH, low-dose UFH, VKA, fondaparinux, aspirin (all Grade 1B), or an intermittent pneumatic compression device (IPCD) (Grade 1C) for VTE prophylaxis, whereas it does not recommend using apixaban, rivaroxaban, or dabigatran [1]. The duration of pharmacological VTE prophylaxis should continue for 28-35 days postoperatively [1]. Jeong et al. [28] compared the clinical efficacy and side-effect profiles of aspirin, dextran 40, and LMWH in preventing thromboembolic phenomena after hip fracture surgery. Among the three pharmacologic agents, they found a low incidence of thromboembolic phenomena, $\mathrm{PE}$, and fatal $\mathrm{PE}$, with no difference in thromboembolic prophylaxis efficacy [28].

Thus, a personalized protocol for pharmacological VTE prophylaxis should be used based on the surgical plan, underlying condition, and risk of thrombosis in each patient.

\section{Should pharmacological prophylaxis be administered in all Asian patients who undergo knee and hip arthroplasty and hip fracture surgery? Recommendation}

In Asian patients with elevated VTE risk who are undergoing knee and hip arthroplasty or hip fracture surgery, a pharmacological prophylaxis should be administered, except in patients with increased bleeding risk or contraindications for pharmacological prophylaxis.

Delegate vote: Agree 89.0\%, Disagree 5.5\%, Abstain 5.5\% (Strong Consensus)

\section{Justification}

There are several studies to show that Asian ethnicity is associated with a lower DVT and VTE incidence compared to Caucasians [29-32]. However, in all patients with elevated VTE risk, regardless of ethnicity, the pharmacological prophylaxis is recommended [1, 3, 33, 34].

Although there is no validated risk assessment model applied for Asians, there are no studies to show that Asians have a higher bleeding risk than Caucasians after surgery in the presence of anticoagulants such as UFH, LMWH, Fondaparinux, and the new oral anticoagulants. Liew et al. [34] have proposed using the Caprini risk score for VTE prevention in Asians. A mechanical VTE prophylaxis is suitable in patients with a high risk of bleeding [35].

According to the current guidelines, the type of pharmacological prophylaxis should be considered following risk stratification [3]. A pharmacological prophylaxis is contraindicated in active bleeding and untreated congenital coagulopathies, and only an IPCD is recommended for this group of patients [36]. Contraindications for the IPCD include acute thrombophlebitis, suspected DVT, congestive heart failure, pulmonary edema, and leg ischemia due to peripheral vascular disease $[3,33]$.

In conclusion, a combination of pharmacological and mechanical VTE prophylaxis is recommended for Asian patients who have elevated VTE risk, based on risk stratification. A mechanical prophylaxis could be considered to use alone in patients who have increased bleeding risk.

\section{Considering VTE risk and bleeding risk, which method of VTE prophylaxis should apply for Asian patients undergoing knee and hip arthroplasty or hip fracture surgery? \\ 4A. Standard VTE risk without bleeding risk}

Recommendation: Mechanical prophylaxis alone or pharmacological prophylaxis combined with mechanical prophylaxis

Delegate vote: Agree 97.2\%, Disagree 1.4\%, Abstain $1.4 \%$ (Strong Consensus)

\section{B. Elevated VTE risk without bleeding risk}

Recommendation: Pharmacological prophylaxis combined with mechanical prophylaxis

Delegate vote: Agree 98.6\%, Disagree 0\%, Abstain 1.4\% (Strong Consensus). 


\section{C. Standard VTE risk with bleeding risk}

Recommendation: Mechanical prophylaxis alone

Delegate vote: Agree 97.2\%, Disagree 1.4\%, Abstain 1.4\% (Strong Consensus)

\section{D. Elevated VTE risk with bleeding risk}

Recommendation: Mechanical prophylaxis alone or combined with aspirin

Delegate vote: Agree 86.3\%, Disagree 8.2\%, Abstain 5.5\% (Strong Consensus)

\section{Justification}

The 2008 AAOS guidelines suggest that mechanical prophylaxis has no bleeding risk [37]. Therefore, mechanical prophylaxis methods should remain in place regardless of VTE risk or bleeding risk [37].

The 2018 European guidelines on perioperative VTE prophylaxis suggest the use of aspirin for VTE prevention after THA, TKA, and hip fracture surgery in patients with an increased bleeding risk (Grade 2C) [38].

According to the 2012 ACCP guidelines, it is recommended to use dual prophylaxis with an antithrombotic agent and an IPCD during the hospital stay in patients undergoing major orthopedic surgery (Grade 2C) [1].

The 2018 National Institute for Health and Care Excellence (NICE) guideline recommended using antiembolism stockings until discharge, combined with LMWH, as an option in patients undergoing elective TKA and THA [39]. For hip fracture, it is recommended to consider IPCD at the time of admission if pharmacological prophylaxis is contraindicated [39]. This should be continued until the patient no longer has significantly reduced mobility [39].

According to the American Society of Hematology (ASH) 2019 guideline, depending on the risk of VTE and bleeding based on the individual patient and the type of surgical procedure, it is recommended to use combined prophylaxis or mechanical prophylaxis alone [36]. For patients considered at high risk of bleeding, the balance of effects may favor mechanical methods over pharmacological prophylaxis [3]. For patients considered at high risk of VTE, combined prophylaxis is favored over mechanical or pharmacological prophylaxis alone [26].

The Scottish Intercollegiate Guidelines Network (SIGN) guideline suggested that patients with increased risk of bleeding should be given mechanical prophylaxis alone [40]. Pneumatic foot pumps can be considered for prophylaxis as an alternative to IPCD in orthopedic surgery patients [40]. However, the guideline did not recommend combined mechanical with pharmacological prophylaxis [40].

A recent study in Singapore showed a low prevalence of VTE using mechanical prophylaxis alone without chemoprophylaxis in patients who underwent TKA. The prevalence of significant VTE was only $0.82 \%$ [29]. With proper patient selection, risk stratification, and stringent perioperative protocols, mechanical prophylaxis alone without routine chemoprophylaxis may be enough in Asians undergoing TKA [29].

A systematic review and meta-analysis of 1399 patients from six RCTs concluded that the addition of IPCD with pharmacological prophylaxis had benefits in DVT prevention in patients undergoing both knee and hip replacement [41]. In TKA, the rate of DVT was significantly reduced from $18.7 \%$ with anticoagulation alone to $3.7 \%$ with combined modalities [41]. In THA, the rate of DVT was reduced from $9.7 \%$ with anticoagulation alone to $0.9 \%$ with additional mechanical compression, and the rate of DVT was reduced from $8.7 \%$ with mechanical compression alone to $7.2 \%$ with additional pharmacological prophylaxis but was not significant [41]. By contrast, the incidence of PE could not be interpreted [41].

In an Asian study, an RCT of Chinese patients, the patients were randomized into four groups to receive graduated compression stockings (GCSs) alone (group A), GCS + LMWH (group B), GCS + IPCD (group C), and GCS + IPCD + LMWH (group D) [42]. The overall incidence of DVT was 5.1\% [42]. Group A had the highest incidence of DVT (8.8\%), followed by group C (5.2\%), group B (3.8\%), and group D (2.6\%) [42]. There was a significant difference in the incidence of DVT between groups A and D [42]. The incidence of DVT was significantly lower in LMWH-treated patients (group B and group D) than in non-LMWH-treated patients (group A and group C) [42]. The authors concluded that a combined mechanical and pharmacological VTE prophylaxis showed better effectiveness of VTE prevention than mechanical or pharmacological prophylaxis alone [42].

A systematic review of major orthopedic surgeries showed that LMWH with a mechanical device had a significantly decreased risk of total DVT compared to LMWH alone in patients undergoing THA [43]. In patients with TKA, the antiplatelet with mechanical device group had a lower incidence of total DVT than the group in which antiplatelets alone were used [43].

In a case series of 38 patients who underwent lower extremity orthopedic surgery with the highest risk of both venous thrombosis and bleeding, a portable pneumatic compression device was used to prevent VTE [44]. The results showed that the incidence of asymptomatic DVT was $5.3 \%$, and that of symptomatic DVT was $2.6 \%$ [44]. No major bleeding or adverse events were observed [44]. Also, a retrospective study reviewed all patients with hemophilia A or B who underwent primary TKA and THA using a mechanical prophylaxis without chemoprophylaxis [45]. They found that there was only one hemophilia B patient with clinically significant VTE, 
for an incidence of 1.02\% [45]. Perez Botero et al. [46] reported similar results in a review of patients with hemophilia A or B who underwent 71 TKAs or THAs. Compression stockings were applied to all patients, of whom 10.5\% had IPCDs, and 2.5\% had LMWH [46]. Only one patient who received LMWH had asymptomatic DVT [46]. The incidence of symptomatic VTE was $0.5 \%$ [46]. The authors concluded that hemophilia patients who had a high risk of bleeding could safely use mechanical prophylaxis of DVT alone without chemoprophylaxis [46].

Bleeding risks, such as those caused by acute liver failure, concurrent use of anticoagulants, lumbar puncture/ epidural/spinal anesthesia expected within the next $12 \mathrm{~h}$, lumbar puncture/epidural/spinal anesthesia within the previous $4 \mathrm{~h}$, acute stroke, thrombocytopenia, uncontrolled systolic hypertension, or untreated inherited bleeding disorders, are a contraindication for anticoagulants $[3,33]$. Previous major bleeding, severe renal failure, concomitant antiplatelet agent, extensive surgical dissection, and revision surgery are general risk factors for bleeding [3, 33]. In patients with bleeding risk, an IPCD is indicated [3,33].

\section{Do the dosage and the duration of perioperative tranexamic acid (TXA) affect the pharmacological VTE prophylaxis in patients undergoing knee and hip arthroplasty and hip fracture surgery? Recommendation}

No. TXA administration, at the dosage and the duration administered in knee and hip arthroplasty and hip fracture surgery, is not associated with elevated VTE risk for patients without a known history of VTE.

Delegate vote: Agree 95.9\%, Disagree 0\%, Abstain 4.1\% (Strong Consensus)

\section{Justification}

There is still no conclusive evidence that TXA increases the risk of VTE [47]. Administration of oral, topical, or intravenous TXA in patients without a known history of VTE does not increase the risk of developing VTE compared to placebo during the perioperative episode of a primary TJA [47]. A meta-analysis investigating the impact of TXA administration on the risk of VTE included 77 high-quality and one moderate-quality RCT [47]. Almost all of the studies excluded patients with a history of a thromboembolic event [47]. In RCTs on knee and hip arthroplasty, the results demonstrated no difference in the VTE rate in the TXA group compared with those in the placebo group [47]. Moreover, the clinical practice guideline of the American Association of Hip and Knee Surgeons (AAHKS) gives a "strong" recommendation that the administration of TXA does not increase risk of VTE [48].
For hip fracture surgery, the meta-analysis of 11 RCTs in 892 patients found that DVT significantly occurred in 16 of 423 patients (3.8\%) in the TXA group compared with 8 of 431 patients (1.9\%) in the control group. This showed that the risk of DVT was similar in both groups, which means that the use of TXA in hip fracture surgery is safe and does not increase the risk for VTE [49].

\section{Does pharmacological VTE prophylaxis increase the incidence of wound drainage and/or infection in patients undergoing knee and hip arthroplasty and hip fracture surgery? Recommendation}

Yes, pharmacological VTE prophylaxis may increase wound drainage. Prolonged wound drainage was reported as a significant predictor of wound infection after THA. However, there is no evidence that anticoagulants for VTE prophylaxis increase the risk of infection in TKA and hip fracture surgery.

Delegate vote: Agree 98.6\%, Disagree 1.4\%, Abstain 0\% (Strong Consensus)

\section{Justification}

Pharmacological VTE prophylaxis may increase bleeding, leading to increased wound drainage from a suction catheter, and can also lead to increased subcutaneous wound bleeding and an increase in surgical wound drainage from a surgical wound [50]. Patel et al. [50] conducted a retrospective study of 1211 THA patients and 1226 TKA patients. They found that prophylaxis against VTE with LMWH was associated with a significant increase in wound drainage after THA but not after TKA [50]. Also, there was prolonged wound drainage in the group treated with LMWH compared to the groups treated with Coumadin (warfarin) or aspirin and mechanical compression [50]. On the fifth day after the operation, the LMWH group had significantly more wound drainage than the aspirin group [50]. Moreover, there was a significantly strong positive correlation between the length of hospital stay and the number of days until the surgical wound was dry [50]. The correlation was significantly stronger in the THA group compared to the TKA group [50]. Prolonged wound drainage was a significant predictor of wound infection after THA, and each day of prolonged drainage was associated with a $42 \%$ increase in the risk of wound infection [50]. However, prolonged wound drainage did not increase the risk of infection in TKA [50].

Agaba et al. [51] conducted a cohort study of VTE prophylaxis in THA. They found that warfarin was associated with the highest number of postoperative complications at 30 days following surgery [51]. The complications of warfarin consisted of incision and drainage (I\&D; odds ratio [OR], 2.04), hematoma (OR, 
1.95), transfusion (OR, 2.29), PE (OR, 1.72), DVT (OR, 1.53), prosthetic joint infection (PII; OR, 1.44), and hemorrhage (OR, 1.92) [51]. The apixaban complications were hematoma (OR, 4.0) and hemorrhage (OR, 3.59) during the 30 days following surgery [51]. There were no statistically significant complications associated with enoxaparin, rivaroxaban, or fondaparinux during the 30day postoperative period [51].

Runner et al. [52] studied databases from 2014 to 2016 in a total of 22,072 primary TJA cases. The study showed that patients receiving prophylaxis with aspirin or a sequential compression device were significantly associated with having no increased complications [52]. The use of prophylaxis with heparin, enoxaparin, warfarin, rivaroxaban, fondaparinux, and all other prophylactic strategies was significantly associated with a higher likelihood of mild thrombosis $(0.9 \%$ vs. $0.2 \%)$, mild bleeding ( $1.3 \%$ vs. $0.4 \%)$, moderate thrombosis $(1.2 \%$ vs. $0.4 \%)$, moderate bleeding ( $2.7 \%$ vs. $2.1 \%)$, severe bleeding events $(1.2 \%$ vs. $0.9 \%)$, infection $(1.9 \%$ vs., $1.3 \%)$, and death within 90 days ( $0.7 \%$ vs. $0.3 \%$ ) [52].

Prolonged wound drainage associated with anticoagulation following THA or TKA has been associated with infection and increased length of hospital stay [50, 52]. Previous studies have investigated the risk of bleeding, prolonged wound drainage, and length of hospital stay among current medications, such as aspirin, LMWH, warfarin, rivaroxaban, and fondaparinux, and have not find significant differences in these complications [50, 52]. However, a retrospective study has shown that, except for aspirin, all other chemoprophylaxis agents increase the risk of bleeding [52].

\section{A. Among all available pharmacological agents for VTE prophylaxis, which is the most appropriate for Asians undergoing elective knee and hip arthroplasty? Recommendation}

Aspirin is the most appropriate agent for patients with standard VTE risk. Direct oral anticoagulants (DOACs) or LMWH are the most appropriate agents for patients with elevated VTE risk.

Delegate vote: Agree 82.2\%, Disagree 8.2\%, Abstain 9.6\% (Strong Consensus)

\section{B. Among all available pharmacological agents for VTE prophylaxis, which is the most appropriate for Asians undergoing hip fracture surgery? \\ Recommendation}

Inconclusive; there is not enough evidence to support the most appropriate agents.

Delegate vote: Agree 93.2\%, Disagree 4.1\%, Abstain 2.7\% (Strong Consensus)

\section{Justification}

According to the ACCP guideline released in 2012, for patients undergoing TKA or THA, it is recommended to use one of the following: $\mathrm{LMWH}$, fondaparinux, apixaban, dabigatran, rivaroxaban, UFH, VKA, aspirin (all Grade 1B) or an IPCD (Grade 1C) [1]. It is recommended that patients undergoing hip fracture surgery use one of the following: LMWH, fondaparinux, UFH, VKA, aspirin (all Grade 1B) or an IPCD (Grade 1C) [1]. The guideline also recommended using LMWH in preference to the other agents for patients undergoing TKA and THA and hip fracture surgery (Grade 2B) [1].

The NICE guidelines were released in 2018 and recommended aspirin, $\mathrm{LMWH}$, or rivaroxaban for patients undergoing elective knee replacement [39]. For patients undergoing elective hip replacement, the guidelines recommended LMWH or rivaroxaban [39]. For patients undergoing hip fracture surgery, LMWH or fondaparinux was recommended [39].

The ASH guidelines, released in 2019, recommended using aspirin or anticoagulants for patients undergoing TKA or THA [36]. If the anticoagulants are selected, it is recommended to use DOACs over LMWH [36]. If DOACs are not selected, it is recommended to use LMWH rather than warfarin [36]. For patients undergoing hip fracture repair, one should use LMWH or UFH [36].

Although a study from Singapore showed a low prevalence of VTE in patients undergoing TKA without chemoprophylaxis [29], aspirin could be an option for pharmacological prophylaxis in elective knee and hip arthroplasty, which was recommended by the ACCP, NICE, and ASH guidelines. Several meta-analyses suggested that aspirin is safe and shows good efficacy following TJA and that it demonstrated noninferiority to other anticoagulants [2, 53-56]. The relative risk (RR) of VTE after TKA and THA was 1.12 for aspirin compared with other anticoagulants [56]. Comparable findings were observed for DVT (RR, 1.04) and PE (RR, 1.01) [56]. The risk of adverse events, including major bleeding, wound hematoma, and wound infection, was not statistically significantly different in patients receiving aspirin vs. other anticoagulants [56]. When analyzing TKAs and THAs separately, there was no statistically significant difference in the risk of VTE, DVT, or PE between aspirin and other anticoagulants [56]. Aspirin had a VTE risk not statistically significantly different from that of LMWH or rivaroxaban [56]. Aspirin is an inexpensive, widely available, and well-tolerated agent that does not require routine blood tests and causes fewer adverse events, such as hematoma [56]. Because of the lower prevalence of VTE in Asians, with several studies supporting the use of aspirin, it is reasonable to use aspirin as the pharmacological VTE prophylaxis in those who are considered as having a standard VTE risk. 
For Asian patients who have elevated VTE risk and undergo elective knee and hip arthroplasty, some studies favored using aspirin [55]. According to the retrospective study of Tan et al. [55] on 60,467 joint arthroplasties, patients were considered high risk by score $>70$ points using the VTE calculator described by Huang et al. [57] The authors concluded that the use of warfarin or LMWH in higher-risk patients did not necessarily result in a reduction in symptomatic VTE [57]. By contrast, aspirin administered to higher-risk patients seemed to be as effective as potent anticoagulants and more effective than warfarin [55]. The study of Huang et al. [57] in 30, 270 patients concluded that aspirin is as effective as and safer than warfarin for VTE prophylaxis after TJA, even in patients at higher risk of VTE. However, because of the limited evidence of using aspirin for VTE prophylaxis in TKA and THA, it is reasonable that more potent anticoagulants should be considered rather than aspirin for patients with elevated VTE risk.

In patients with hip fractures, immobility has been reported to increase the risk of VTE [58]. Patients with hip fractures should be classified as elevated-risk patients [58]. Although aspirin was only recommended in hip fracture surgery by the ACCP guideline, other guidelines favored other anticoagulant drugs [1]. LMWH was recommended by the ACCP, NICE, ASH, and SIGN guidelines. The ACCP recommended DOACs in elective knee and hip arthroplasty, but not in hip fracture surgery; however, the NICE, ASH, and SIGN guidelines preferred DOACs over LMWH $[1,36,39,40,59]$. As most guidelines and systematic reviews supported using anticoagulants rather than aspirin in patients who undergo hip fracture surgery, it is reasonable that more potent anticoagulants than aspirin should be used for this group of patients.

\section{Which pharmacological agents for VTE prophylaxis are the most cost-effective for Asian patients undergoing knee and hip arthroplasty? \\ Recommendation}

In patients without elevated risk for VTE, aspirin is a more cost-effective prophylactic agent than other agents.

Delegate vote: Agree 90.4\%, Disagree 1.4\%, Abstain 8.2\% (Strong Consensus)

\section{Justification}

Mostafavi et al. compared the cost and health benefits of anticoagulation using warfarin or aspirin following TJA and demonstrated that the use of aspirin provided a higher quality-adjusted life year (QALY) measure and lower cost than warfarin in all ages for both TKA and THA [60]. Schousboe et al. compared the costeffectiveness of LMWH or 160-mg aspirin for VTE prophylaxis after TJA and concluded that aspirin is a cost-effective choice for VTE prophylaxis following THA for patients with no history of VTE [5]. The preferred choice following TKA depends on age and is uncertain for those younger than 80 years old [5]. Dawoud et al. [61] compared 15 VTE prophylaxis strategies in elective THA with 12 VTE prophylaxis strategies in elective TKA. They concluded that a strategy consisting of LMWH for ten days, followed by aspirin for 28 days, was the most cost-effective for elective THA [61]. A footpump strategy followed closely by aspirin (low dose) was the most cost-effective for elective TKA [61].

Based on these three studies, it seems that aspirin is a cost-effective VTE prophylaxis agent and can be used safely in patients without a history of VTE who are at low risk for the development of VTE following knee or hip arthroplasty. Although these three studies did not investigate Asian patients, the Asia-Pacific VTE consensus experts agreed that a similar recommendation could apply to Asian patients.

\section{In Asian patients, how long should a pharmacological prophylaxis be given for knee and hip arthroplasty and hip fracture surgery? \\ Recommendation}

Although the optimal duration of pharmacological prophylaxis remains inconclusive, the recommended minimum duration should be 10-14 days.

Delegate vote: Agree 83.6\%, Disagree 9.6\%, Abstain 6.8\% (Strong Consensus)

\section{Justification}

The NICE guideline, released in 2018, recommended pharmacological prophylaxis for 14 days in TKA, 28 days in THA, and one month in hip fracture surgery [39].

The ASH guideline, released in 2019, recommended extended antithrombotic prophylaxis over short-term antithrombotic prophylaxis for patients undergoing major surgery (not specific only to orthopedic surgery) [36]. Extended prophylaxis was considered as beyond 3 weeks, and short-term prophylaxis was considered as up to 2 weeks [36].

The SIGN guideline, released in 2010, recommended extended pharmacological prophylaxis for orthopedic surgery (recommendation Grade A) [40]. However, the optimal duration of extended prophylaxis is unclear [40]. The benefit of post-discharge extended prophylaxis with LMWH is higher in THA than in TKA patients [40].

A systematic review comparing prolonged-duration (21 days) with standard-duration (7-10 days) thromboprophylaxis demonstrated that, in THA, there were fewer symptomatic VTE, PE, nonfatal PE, DVT, asymptomatic DVT, and proximal DVT events with prolonged-duration than with standard-duration prophylaxis [62]. In hip fracture surgery, patients who received 
prolonged prophylaxis had fewer symptomatic objectively confirmed VTE, DVT, proximal DVT, and distal DVT events than those who received standard-duration prophylaxis [62]. However, in TKA, there were no statistically significant differences in any reported outcomes between prolonged-duration prophylaxis and standardduration prophylaxis [62].

A prospective study with 197 patients who were undergoing elective TKA and THA and having extended LMWH with mechanical prophylaxis for 4 weeks, compared with a historical group of 795 patients with shortterm thromboprophylaxis for only 7-11 days, concluded that extended thromboprophylaxis was more effective than short-term prophylaxis [63].

Parvizi et al. showed that the highest prevalence of symptomatic VTE occurs 1 week after TJA. In detail, $81 \%$ occurred within three postoperative days, $89 \%$ within 1 postoperative week, and $94 \%$ within 2 postoperative weeks [64]. They recommended continuing prophylaxis until the end of these periods [64].

By contrast, two recent studies demonstrated different outcomes $[65,66]$. The first study, in 2015, a retrospective review of all primary THAs from the National Joint Registry of Denmark, demonstrated that the 90-day risks of VTE were $1.1 \%$ (short), 1.4\% (standard), and 1.0\% (extended) and the risk of major bleeding was $1.1 \%$ (short), $1.0 \%$ (standard), and $0.7 \%$ (extended) [66]. The study concluded that there was no difference in the risks of symptomatic VTE, VTE/death, or bleeding concerning thromboprophylaxis duration [66]. The second study, in 2019, was a retrospective study of all primary THAs from the Nordic Arthroplasty Register Association (NARA), divided into three groups: short (1-5 days), standard (6-14 days), and extended ( 215 days) duration of thromboprophylaxis [65]. The study demonstrated that the 90-day cumulative incidence of VTE was $1.0 \%$ for patients with standard treatment, 1.1\% for those with short-term treatment, and $1.0 \%$ for those with extended treatment [65]. In conclusion, from these two studies, short-duration prophylaxis had comparable effectiveness to extended-duration prophylaxis in THA $[65,66]$. However, these evidences were weak because of the observational study designs from the National Joint Registry.

\section{What is the optimal dose, starting time, and duration of aspirin usage for VTE prophylaxis in Asian patients who undergo knee \& hip arthroplasty? \\ Recommendation}

Low dose aspirin ranging from $81-162 \mathrm{mg} /$ day is sufficient for VTE prophylaxis. However, the starting time of administration and duration of usage of aspirin remains inconclusive.

Delegate vote: Agree 97.3\%, Disagree 0\%, Abstain 2.7\% (Strong Consensus)

\section{Justification}

A recent systematic review categorized low-dose and high dose of aspirin prophylaxis for VTE based on $162 \mathrm{mg}$ of aspirin use [67]. In the low dose group, dosages of aspirin ranged from $75 \mathrm{mg}$ daily to $160 \mathrm{mg}$ daily [67]. In the low dose group, two studies looked at Asian patients $[68,69]$. These two studies were done in China, using aspirin $100 \mathrm{mg}$ daily $[68,69]$. Both studies demonstrated that aspirin $100 \mathrm{mg}$ daily had a comparable effect for VTE prophylaxis as LMWH $[68,69]$.

There were no significant differences in symptomatic PE, symptomatic DVT, 90-day mortality, or major bleeding between patients receiving low-dose or high-dose aspirin for the entire systematic review [67]. Compared with warfarin, there was also a significantly higher risk of symptomatic DVT with warfarin compared to lowdose aspirin [67]. The studies included in this systematic review reported various durations of aspirin prophylaxis ranging from 14 days to 6 weeks [67]. They found no significant difference between incidences of PE or DVT and the different durations of aspirin treatment examined ( $<4$ weeks, four weeks, and $>4$ weeks) [67].

Three studies compared low dose aspirin with high dose aspirin directly in the same population [70-72]. Faour et al. investigated the aspirin prophylaxis for VTE in 2018, and 2019 [70, 71]. The first study included patients with TKA and compared between the low dose group (81mg, twice a day) and high dose group $(325 \mathrm{mg}$, twice a day [71]. Also, all patients received pneumatic compression stockings, the duration of treatment was 46 weeks [71]. Regression model showed no correlation between aspirin dose and VTE incidence or DVT [71]. The incidence of $\mathrm{PE}$ was $0.2 \%$ in the high-dose aspirin group compared with $0.4 \%$ in the low-dose aspirin group without a significant difference [71]. Bleeding and 90day mortality was similar between the groups [71]. The second retrospective study in THA patients with lowdose aspirin $81 \mathrm{mg}$ twice a day, high-dose aspirin 325 mg twice a day and 4- to 6-week duration of treatment [70]. After accounting for confounders, regression analyses showed no difference between aspirin doses and the 90-day incidence of symptomatic VTE or symptomatic DVT [70]. Bleeding and 90-day mortality was not different between the groups [70]. In conclusion of these two studies, low-dose aspirin was not inferior to highdose aspirin for the prevention of VTE after TKA and THA [70, 71]. Parvizi et al [72]. in 2017 performed prospective crossover study including 4,651 patients for aspirin prophylaxis of VTE for 4 weeks based on different dosage of aspirin. The low dose group received $81 \mathrm{mg}$ of aspirin twice a day and the high dose group received $325 \mathrm{mg}$ of aspirin twice a day.. The incidence of VTE of $0.1 \%$ in the $81 \mathrm{mg}$ aspirin group was not significantly different from $0.3 \%$ in the $325 \mathrm{mg}$ aspirin group [72]. 
The incidence of gastrointestinal bleeding or ulceration of $0.3 \%$ in the $81 \mathrm{mg}$ aspirin group was slightly, but not significantly lower than the $0.4 \%$ in the $325 \mathrm{mg}$ aspirin group [72]. The incidence of acute periprosthetic joint infection was $0.2 \%$ in the $81 \mathrm{mg}$ aspirin group compared with $0.5 \%$ in the $325 \mathrm{mg}$ aspirin group [72]. The 90-day mortality rate was the same in both groups at $0.1 \%$ in the $81 \mathrm{mg}$ aspirin group and $0.1 \%$ in the $325 \mathrm{mg}$ aspirin group [72].

In conclusion, low dose aspirin ranging from 81-162 $\mathrm{mg} / \mathrm{d}$ is not inferior to high dose aspirin of $325-650 \mathrm{mg} /$ $\mathrm{d}$ for VTE prophylaxis. The duration of aspirin usage has wide variation from 14 days to 6 weeks. However, the evidence available on the optimal time of administration and the optimal duration of aspirin usage for VTE prophylaxis is of limited quality and remains inconclusive.

\section{A. Should the dose and duration of pharmacological} VTE prophylaxis in Asian patients with standard VTE risk be adjusted from the recommended dose and duration by the American or European guidelines?

\section{Recommendation}

There is evidence that a lower dose of the pharmacologic agent is effective, but there is limited evidence for the administration timing. Therefore, a lower dose with delayed pharmacologic administration can apply in Asian patients with standard VTE risk.

Delegate vote: Agree 87.7\%, Disagree 4.1\%, Abstain 8.2\% (Strong Consensus)

\section{B. Should the dose and duration of pharmacological VTE prophylaxis in Asian patients with elevated VTE risk be adjusted from the recommended dose and duration by the American or European guidelines? Recommendation}

There is limited evidence that a lower dose and/or delayed administration of the pharmacologic agent is effective in Asian patients with elevated VTE risk. Therefore, the recommended dose and duration should apply in Asian patients with elevated VTE risk.

Delegate vote: Agree 89.0\%, Disagree 5.5\%, Abstain 5.5\% (Strong Consensus)

\section{Justification}

There is evidence demonstrating that Asian patients have lower DVT prevalence than Caucasians. Bin Abd Razak et al. [29] showed the prevalence of VTE without chemoprophylaxis in Singaporean patients who underwent TKA and mechanical prophylaxis alone. The prevalence of significant VTE is $0.82 \%$, which is significantly lower than that for Caucasians [29]. Fuji et al. [73] found that the mean body weight of Japanese TKA and THA patients who participated in their study was approximately two-thirds that of their Caucasian counterparts. They adjusted their DVT prophylaxis regimen by decreasing the enoxaparin dose to $20 \mathrm{mg}$ bid and confirmed that it is the proper regimen in Japanese patients [73].

Mihara et al. [74] performed a retrospective study of DVT prophylaxis using low-dose aspirin in patients who underwent THA. The patients were divided into a lowrisk VTE group and a high-risk VTE group [74]. Lowrisk patients received aspirin for 28 days postoperatively [74]. High-risk patients, such as those diagnosed with obesity and/or with a history of VTE, received anticoagulants (enoxaparin or edoxaban) for five days postoperatively, followed by $100 \mathrm{mg} /$ day of aspirin for 28 days [74]. There was a low incidence of symptomatic DVT and PE [74]. There was no postoperative fatal bleeding or bleeding from any organ, such as gastrointestinal or cerebral hemorrhage [74]. This study concluded that the hospital's risk-stratified protocol using low-dose aspirin or anticoagulants effectively prevented symptomatic VTE [74]. These results were better than those reported from Western countries [74]. There are two studies from China using $100 \mathrm{mg}$ of aspirin daily [67, 68]. Both studies demonstrated that taking $100 \mathrm{mg}$ of aspirin daily had a comparable effect for VTE prophylaxis as LMWH $[67,68]$.

Therefore, the usual dose and duration in knee or hip arthroplasty may not be necessary in Asians at standard risk for VTE because of their lower rate of VTE and lower body weight compared to Caucasians.

\section{Abbreviations \\ AAHKS: American Association of Hip and Knee Surgeons; AAOS: American Academy of Orthopaedic Surgeons; ACCP: American College of Chest Physicians; ASH: American Society of Hematology; DOAC: Direct oral anticoagulant; DTI: Direct thrombin inhibitor; DVT: Deep vein thrombosis; FDA: Food and Drug Administration; INR: International normalized ratio; IPCD: Intermittent pneumatic compression device; LMWH: Low-molecular- weight heparin; NICE: National Institute for Health and Care Excellence; PE: Pulmonary embolism; QALY: Quality-adjusted life year; RCT: Randomized controlled trial; THA: Total hip arthroplasty; TJA: Total joint arthroplasty; TKA: Total knee arthroplasty; TXA: Tranexamic acid; UFH: Unfractionated heparin; VKA: Vitamin K antagonist; VTE: Venous thromboembolism}

\section{Acknowledgements}

The consensus group especially thanks Western professors William J. Maloney and Christopher S. Mow, who are leading experts in the field of total joint arthroplasty and related complications, for their advice and dedication regarding the current study. NOTE: Members of Asia-Pacific (AP) Region Venous Thromboembolism (VTE) Consensus Group include: Aasis Unnanuntana, Alvin Tan, Anthony Pohl, Apisak Angsugomutkul, Apisit Patamarat, Arak Limtrakul, Aree Tanavalee, Azhar Merican, Azlina Abbas, Badrul Shah Badaruddin, Boonchana Pongcharoen, Bui Hong Thien Khanh, Chaithavat Ngarmukos, Charlee Sumettavanich, Chavanont Sumanasrethakul, Chavarin Amarase, Chee-Ken Chan, Cheng-Fong Chen, Chong Bum Chang, Christopher Scott Mow, Chumroonkiet Leelasestaporn, Chun Hoi Yan, DangKhoa Tran, David Campbell, David Liu, Edi Mustamsir, Edsel Fernandez Arandia, Eun Kyoo Song, G Ruslan Nazaruddin Simanjuntak, Hirotsugu Muratsu, Hyonmin Choe, Jamal Azmi Mohammad, Jason Chi Ho Fan, Ji Hoon Bae, Ji-Wan Kim, Jose Antonio San Juan, Jose Fernando C Syquia, Jun-Ho Kim, KiKi Novito, Kriskamol Sithitool, Manoon Sakdinakiattikoon, Masaaki Matsubara, Mel S Lee, Mohamad Zaim Chilmi, Myint Thaung, Myung Chul 
Lee, Narathorn Kongsakpaisal, Ngai Nung Lo, Nikom Noree, Nobuhiko Sugano, Paphon Sa-ngasoongsong, Pariwat Taweekitikul, Peter Bernardo, Piti Rattanaprichavej, Piya Pinsornsak, Po-Kuei Wu, Pongsak Yuktanandana, Pruk Chaiyakit, Rahat Jarayabhand, Ross W Crawford, Ryuji Nagamine, Saradej Khuangsirikul, Seng Jin Yeo, Siwadol Wongsak, Srihatach Ngarmukos, Sukit Saengnipanthkul, Supparurk Suksumran, Surapoj Meknavin, Thakrit Chompoosang, Than Win, Thana Narinsorasak, Thana Turajane, Thanainit Chotanaphuti, Thanarat Reancharoen, Tokifumi Majima, Ukrit Chaweewannakorn, Viroj Kawinwonggowit, Viroj Larbpaiboonpong, Wanshou Guo, Weerachai Kosuwon, Wei Chai, William J. Maloney, Yee Hong Teo, Yixin Zhou, Yunsu Chen, Yutaka Inaba, Yutthana Khanasuk.

\section{Authors' contributions}

All authors (ST, NB, CT, ST, RMS, CL, and KIK) were involved in study conception and manuscript writing. The corresponding author (KIK) supervised the work. The author(s) read and approved the final manuscript.

\section{Funding}

None.

\section{Availability of data and materials}

Not applicable.

\section{Declarations}

\section{Ethics approval and consent to participate}

Not applicable.

\section{Consent for publication}

Not applicable.

\section{Competing interests}

The authors declare that they have no competing interests.

\section{Author details}

${ }^{1}$ Department of Orthopaedic Surgery, Faculty of Medicine Vajira Hospital, Navamindradhiraj University, Dusit, Bangkok, Thailand. ${ }^{2}$ Nicolaas Institute of Constructive Orthopaedics Research and Education Foundation for Arthroplasty and Sports Medicine, Medistra Hospital, Jakarta, Indonesia. ${ }^{3}$ Department of Orthopaedic Surgery, Faculty of Medicine, Chulalongkorn University and King Chulalongkorn Memorial Hospital, Thai Red Cross Society, Bangkok, Thailand. ${ }^{4}$ Department of Orthopaedic Surgery, King Chulalongkorn Memorial Hospital, Bangkok, Thailand. ${ }^{5}$ Department of Orthopaedics, Nepean Hospital, Penrith, New South Wales, Australia. ${ }^{6}$ Department of Orthopaedics, First Affiliated Hospital of Xinjiang Medical University, Xinjiang, China. ${ }^{7}$ Department of Orthopaedic Surgery, Center for Joint Diseases, Kyung Hee University Hospital at Gangdong, 892 Dongnam-ro, Gangdong-gu, Seoul 134-727, South Korea. ${ }^{8}$ Department of Orthopaedic Surgery, School of Medicine, Kyung Hee University, Seoul, South Korea.

\section{Received: 15 March 2021 Accepted: 4 April 2021}

\section{Published online: 12 August 2021}

\section{References}

1. Falck-Ytter $Y$, Francis CW, Johanson NA, Curley C, Dahl OE, Schulman S, Ortel TL, Pauker SG, Colwell CW Jr (2012) Prevention of VTE in orthopedic surgery patients: Antithrombotic Therapy and Prevention of Thrombosis, 9th ed: American College of Chest Physicians Evidence-Based Clinical Practice Guidelines. Chest 141(2):e278S-e325S. https://doi.org/10.1378/chest.11-2404

2. An W, Phan K, Levy YD, Bruce WJ (2016) Aspirin as thromboprophylaxis in hip and knee arthroplasty: a systematic review and meta-analysis. J Arthroplast 31(11):2608-2616. https://doi.org/10.1016/j.arth.2016.04.004

3. Mont MA, Jacobs JJ (2011) AAOS clinical practice guideline: preventing venous thromboembolic disease in patients undergoing elective hip and knee arthroplasty. J Am Acad Orthop Surg 19(12):777-778. https://doi.org/1 0.5435/00124635-201112000-00008

4. Flevas DA, Megaloikonomos PD, Dimopoulos L, Mitsiokapa E, Koulouvaris $P$, Mavrogenis AF (2018) Thromboembolism prophylaxis in orthopaedics: an update. EFORT Open Rev 3(4):136-148. https://doi.org/10.1302/20585241.3.170018
5. Schousboe JT, Brown GA (2013) Cost-effectiveness of low-molecular-weight heparin compared with aspirin for prophylaxis against venous thromboembolism after total joint arthroplasty. J Bone Joint Surg Am 95(14):1256-1264. https://doi.org/10.2106/JBJS.L.00400

6. Martel N, Lee J, Wells PS (2005) Risk for heparin-induced thrombocytopenia with unfractionated and low-molecular-weight heparin thromboprophylaxis: a meta-analysis. Blood 106(8):2710-2715. https://doi.org/10.1182/blood-2 005-04-1546

7. Kumar A, Talwar A, Farley JF, Muzumdar J, Schommer JC, Balkrishnan R, Wu W (2019) Fondaparinux sodium compared with low-molecular-weight heparins for perioperative surgical thromboprophylaxis: a systematic review and meta-analysis. J Am Heart Assoc 8:e012184

8. Turpie AG, Bauer KA, Eriksson BI, Lassen MR (2002) Fondaparinux vs enoxaparin for the prevention of venous thromboembolism in major orthopedic surgery: a meta-analysis of 4 randomized double-blind studies. Arch Intern Med 162(16):1833-1840. https://doi.org/10.1001/archinte.162.1 6.1833

9. Bauer KA, Eriksson BI, Lassen MR, Turpie AG (2001) Fondaparinux compared with enoxaparin for the prevention of venous thromboembolism after elective major knee surgery. N Engl J Med 345(18):1305-1310. https://doi. org/10.1056/NEJMoa011099

10. Eriksson BI, Borris LC, Friedman RJ, Haas S, Huisman MV, Kakkar AK, Bandel TJ, Beckmann H, Muehlhofer E, Misselwitz F, Geerts W (2008) Rivaroxaban versus enoxaparin for thromboprophylaxis after hip arthroplasty. N Engl J Med 358(26):2765-2775. https://doi.org/10.1056/ NEJMoa0800374

11. Kakkar AK, Brenner B, Dahl OE, Eriksson Bl, Mouret P, Muntz J, Soglian AG, Pap AF, Misselwitz F, Haas S (2008) Extended duration rivaroxaban versus short-term enoxaparin for the prevention of venous thromboembolism after total hip arthroplasty: a double-blind, randomised controlled trial. Lancet 372(9632):31-39. https://doi.org/10.1016/S0140-6736(08)60880-6

12. Lassen MR, Ageno W, Borris LC, Lieberman JR, Rosencher N, Bandel TJ, Misselwitz F, Turpie AG (2008) Rivaroxaban versus enoxaparin for thromboprophylaxis after total knee arthroplasty. N Engl J Med 358(26): 2776-2786. https://doi.org/10.1056/NEJMoa076016

13. Turpie AG, Lassen MR, Davidson BL, Bauer KA, Gent M, Kwong LM, Cushner FD, Lotke PA, Berkowitz SD, Bandel TJ, Benson A, Misselwitz F, Fisher WD (2009) Rivaroxaban versus enoxaparin for thromboprophylaxis after total knee arthroplasty (RECORD4): a randomised trial. Lancet 373(9676):16731680. https://doi.org/10.1016/S0140-6736(09)60734-0

14. Lassen MR, Raskob GE, Gallus A, Pineo G, Chen D, Portman RJ (2009) Apixaban or enoxaparin for thromboprophylaxis after knee replacement. $\mathrm{N}$ Engl J Med 361(6):594-604. https://doi.org/10.1056/NEJMoa0810773

15. Lassen MR, Gallus A, Raskob GE, Pineo G, Chen D, Ramirez LM (2010) Apixaban versus enoxaparin for thromboprophylaxis after hip replacement. N Engl J Med 363(26):2487-2498. https://doi.org/10.1056/NEJMoa1006885

16. Lassen MR, Raskob GE, Gallus A, Pineo G, Chen D, Hornick P (2010) Apixaban versus enoxaparin for thromboprophylaxis after knee replacement (ADVANCE-2): a randomised double-blind trial. Lancet 375(9717):807-815. https://doi.org/10.1016/S0140-6736(09)62125-5

17. Ginsberg JS, Davidson BL, Comp PC, Francis CW, Friedman RJ, Huo MH, Lieberman JR, Muntz JE, Raskob GE, Clements ML, Hantel S, Schnee JM, Caprini JA (2009) Oral thrombin inhibitor dabigatran etexilate vs North American enoxaparin regimen for prevention of venous thromboembolism after knee arthroplasty surgery. J Arthroplast 24(1):1-9. https://doi.org/10.101 6/j.arth.2008.01.132

18. Eriksson BI, Dahl OE, Rosencher N, Kurth AA, van Dijk CN, Frostick SP, Kälebo P, Christiansen AV, Hantel S, Hettiarachchi R, Schnee J, Büller HR (2007) Oral dabigatran etexilate vs. subcutaneous enoxaparin for the prevention of venous thromboembolism after total knee replacement: the RE-MODEL randomized trial. J Thromb Haemost 5(11):2178-2185. https://doi.org/1 0.1111/j.1538-7836.2007.02748.x

19. Eriksson BI, Dahl OE, Rosencher N, Kurth AA, van Dijk CN, Frostick SP, Prins MH, Hettiarachchi R, Hantel S, Schnee J, Büller HR (2007) Dabigatran etexilate versus enoxaparin for prevention of venous thromboembolism after total hip replacement: a randomised, doubleblind, non-inferiority trial. Lancet 370(9591):949-956. https://doi.org/10.1 016/S0140-6736(07)61445-7

20. Eriksson BI, Dahl OE, Huo MH, Kurth AA, Hantel S, Hermansson K, Schnee JM, Friedman RJ (2011) Oral dabigatran versus enoxaparin for thromboprophylaxis after primary total hip arthroplasty (RE-NOVATE I*). A 
randomised, double-blind, non-inferiority trial. Thromb Haemost 105(4):721729. https://doi.org/10.1160/TH10-10-0679

21. Lotke PA, Palevsky H, Keenan AM, Meranze S, Steinberg ME, Ecker ML, Kelley MA (1996) Aspirin and warfarin for thromboembolic disease after total joint arthroplasty. Clin Orthop Relat Res 324:251-258. https://doi.org/10.1097/ 00003086-199603000-00031

22. Heit JA, Berkowitz SD, Bona R, Cabanas V, Corson JD, Elliott CG, Lyons R (1997) Efficacy and safety of low molecular weight heparin (ardeparin sodium) compared to warfarin for the prevention of venous thromboembolism after total knee replacement surgery: a double-blind, dose-ranging study. Ardeparin Arthroplasty Study Group. Thromb Haemost 77:32-38

23. Fitzgerald RH Jr, Spiro TE, Trowbridge AA, Gardiner GA Jr, Whitsett TL, O'Connell MB, Ohar JA, Young TR (2001) Prevention of venous thromboembolic disease following primary total knee arthroplasty. A randomized, multicenter, open-label, parallel-group comparison of enoxaparin and warfarin. J Bone Joint Surg Am 83(6):900-906. https://doi. org/10.2106/00004623-200106000-00012

24. Mismetti P, Laporte S, Zufferey P, Epinat M, Decousus H, Cucherat M (2004) Prevention of venous thromboembolism in orthopedic surgery with vitamin K antagonists: a meta-analysis. J Thromb Haemost 2(7):1058-1070. https:// doi.org/10.1111/j.1538-7836.2004.00757.x

25. Lieberman JR, Pensak MJ (2013) Prevention of venous thromboembolic disease after total hip and knee arthroplasty. J Bone Joint Surg Am 95(19): 1801-1811. https://doi.org/10.2106/JBJS.L.01328

26. Forster R, Stewart M (2016) Anticoagulants (extended duration) for prevention of venous thromboembolism following total hip or knee replacement or hip fracture repair. Cochrane Database Syst Rev 3:Cd004179

27. Kwong LM (2004) Hip fracture and venous thromboembolism in the elderly. J Surg Orthop Adv 13(3):139-148

28. Jeong GK, Gruson Kl, Egol KA, Aharonoff GB, Karp AH, Zuckerman JD, Koval KJ (2007) Thromboprophylaxis after hip fracture: evaluation of 3 pharmacologic agents. Am J Orthop (Belle Mead NJ) 36:135-140

29. Bin Abd Razak HR, Binte Abd Razak NF, Tan HA (2017) Prevalence of venous thromboembolic events is low in Asians after total knee arthroplasty without chemoprophylaxis. J Arthroplast 32(3):974-979. https://doi.org/10.1 016/j.arth.2016.09.008

30. Leizorovicz A, Turpie A, Cohen A, Wong L, Yoo M, Dans A (2005) Epidemiology of venous thromboembolism in Asian patients undergoing major orthopedic surgery without thromboprophylaxis. J Thrombosis Haemostasis 3:28-34

31. Won M-H, Lee G-W, Lee T-J, Moon K-H (2011) Prevalence and risk factors of thromboembolism after joint arthroplasty without chemical thromboprophylaxis in an Asian population. J Arthroplasty 26:11061111

32. Yhim HY, Jang MJ, Bang SM, Kim K, Kim YK, Nam SH, Bae S, Kim SH, Mun YC, Kim I, Jung CW, Oh D (2014) Incidence of venous thromboembolism following major surgery in Korea: from the Health Insurance Review and Assessment Service database. J Thromb Haemost 12:1035-1043

33. Garcia DA, Baglin TP, Weitz Jl, Samama MM (2012) Parenteral anticoagulants: Antithrombotic Therapy and Prevention of Thrombosis, 9th ed: American College of Chest Physicians Evidence-Based Clinical Practice Guidelines. Chest 141(2):e24S-e43S. https://doi.org/10.1378/chest.11-2291

34. Liew NC, Alemany GV, Angchaisuksiri P, Bang SM, Choi G, DA DES, Hong JM, Lee L, Li YJ, Rajamoney GN, Suviraj J, Tan TC, Tse E, Teo LT, Visperas J, Wong RS, Lee LH (2017) Asian venous thromboembolism guidelines: updated recommendations for the prevention of venous thromboembolism. Int Angiol 36(1):1-20. https://doi.org/10.23736/S0392-9590.16.03765-2

35. Leclerc JR, Geerts WH, Desjardins L, Jobin F, Laroche F, Delorme F, Haviernick S, Atkinson S, Bourgouin J (1992) Prevention of deep vein thrombosis after major knee surgery-a randomized, double-blind trial comparing a low molecular weight heparin fragment (enoxaparin) to placebo. Thromb Haemost 67(4):417-423

36. Anderson DR, Morgano GP, Bennett C, Dentali F, Francis CW, Garcia DA, Kahn SR, Rahman M, Rajasekhar A, Rogers FB, Smythe MA, Tikkinen KAO, Yates AJ, Baldeh T, Balduzzi S, Brożek JL, Ikobaltzeta IE, Johal H, Neumann I, Wiercioch W, Yepes-Nuñez JJ, Schünemann HJ, Dahm P (2019) American Society of Hematology 2019 guidelines for management of venous thromboembolism: prevention of venous thromboembolism in surgical hospitalized patients. Blood Adv 3(23):3898-3944. https://doi.org/10.1182/ bloodadvances.2019000975
37. Parvizi J, Azzam K, Rothman RH (2008) Deep venous thrombosis prophylaxis for total joint arthroplasty: American Academy of Orthopaedic Surgeons guidelines. J Arthroplast 23(7):2-5. https://doi.org/10.1016/j.arth.2008.06.028

38. Jenny JY, Pabinger I, Samama CM (2018) European guidelines on perioperative venous thromboembolism prophylaxis: Aspirin. Eur J Anaesthesiol 35(2):123-129. https://doi.org/10.1097/EJA.00000000000000728

39. Gee E (2019) The National VTE Exemplar Centres Network response to implementation of updated NICE guidance: venous thromboembolism in over 16s: reducing the risk of hospital-acquired deep vein thrombosis or pulmonary embolism (NG89). Br J Haematol 186(5):792-793. https://doi. org/10.1111/bjh.16010

40. Network SIGN (2010) Prevention and management of venous thromboembolism. Edinburgh: Healthcare Improvement Scotland

41. Kakkos SK, Warwick D, Nicolaides AN, Stansby GP, Tsolakis IA (2012) Combined (mechanical and pharmacological) modalities for the prevention of venous thromboembolism in joint replacement surgery. J Bone Joint Surg Br 94:729-734

42. Sang CQ, Zhao N, Zhang J, Wang SZ, Guo SL, Li SH, Jiang Y, Li B, Wang JL, Song L, Zhai JJ, Zhang ZY (2018) Different combination strategies for prophylaxis of venous thromboembolism in patients: a prospective multicenter randomized controlled study. Sci Rep 8(1):8277. https://doi. org/10.1038/s41598-018-25274-2

43. Balk EM, Ellis AG, Di M, Adam GP, Trikalinos TA (2017) Venous thromboembolism prophylaxis in major orthopedic surgery: systematic review update. Providence: Brown Evidence-based Practice Center

44. Takahashi Y, Takahira N, Shibuya M, Uchiyama K, Fukushima K, Iwase D, Kawamura T, Miyagi M, Higashiyama R, Moriya M, Sakai K, Tsuda K, Sakamoto M, Akamine A, Takaso M (2020) A portable pneumatic compression device to prevent venous thromboembolism in orthopedic patients with the highest risks of both venous thrombosis and bleeding: a case series study. J Orthop Surg (Hong Kong) 28:2309499020905711

45. Peng HM, Wang LC, Zhai JL, Jiang C, Weng XS, Feng B, Gao N (2019) Incidence of symptomatic venous thromboembolism in patients with hemophilia undergoing hip and knee joint replacement without chemoprophylaxis: a retrospective study. Orthop Surg 11(2):236-240. https://doi.org/10.1111/os.12444

46. Perez Botero J, Spoon DB, Patnaik MS, Ashrani AA, Trousdale RT, Pruthi RK (2015) Incidence of symptomatic venous thromboembolism in patients with hemophilia undergoing joint replacement surgery: a retrospective study. Thromb Res 135(1):109-113. https://doi.org/10.1016/j.thromres.2 014.11.010

47. Fillingham YA, Ramkumar DB, Jevsevar DS, Yates AJ, Shores P, Mullen K, Bini SA, Clarke HD, Schemitsch E, Johnson RL et al (2018) The safety of tranexamic acid in total joint arthroplasty: a direct meta-analysis. J Arthroplasty 33:3070-82.e1

48. Fillingham YA, Ramkumar DB, Jevsevar DS, Yates AJ, Bini SA, Clarke HD, Schemitsch E, Johnson RL, Memtsoudis SG, Sayeed SA, Sah AP, Della Valle CJ (2019) Tranexamic acid in total joint arthroplasty: the endorsed clinical practice guides of the American Association of Hip and Knee Surgeons, American Society of Regional Anesthesia and Pain Medicine, American Academy of Orthopaedic Surgeons, Hip Society, and Knee Society. Reg Anesth Pain Med 44(1):7-11. https://doi.org/10.1136/rapm-2 018-000024

49. Xiao C, Zhang S, Long N, Yu W, Jiang Y (2019) Is intravenous tranexamic acid effective and safe during hip fracture surgery? An updated meta-analysis of randomized controlled trials. Arch Orthop Trauma Surg 139:893-902

50. Patel VP, Walsh M, Sehgal B, Preston C, DeWal H, Di Cesare PE (2007) Factors associated with prolonged wound drainage after primary total hip and knee arthroplasty. J Bone Joint Surg Am 89(1):33-38. https://doi.org/1 0.2106/00004623-200701000-00005

51. Agaba P, Kildow BJ, Dhotar H, Seyler TM, Bolognesi M (2017) Comparison of postoperative complications after total hip arthroplasty among patients receiving aspirin, enoxaparin, warfarin, and factor Xa inhibitors. J Orthop 14(4):537-543. https://doi.org/10.1016/j.jor.2017.08.002

52. Runner RP, Gottschalk MB, Staley CA, Pour AE, Roberson JR (2019) Utilization patterns, efficacy, and complications of venous thromboembolism prophylaxis strategies in primary hip and knee arthroplasty as reported by American Board of Orthopedic Surgery. Part II Candidates. J Arthroplast 34(4):729-734. https://doi.org/10.1016/j.arth.2018.12.015

53. Mistry DA, Chandratreya A, Lee PYF (2017) A systematic review on the use of aspirin in the prevention of deep vein thrombosis in major elective lower 
limb orthopedic surgery: an update from the past 3 years. Surg J (N Y) 3: e191-e1e6

54. Haykal T, Kheiri B, Zayed Y, Barbarawi M, Miran MS, Chahine A, Katato K, Bachuwa (2019) Aspirin for venous thromboembolism prophylaxis after hip or knee arthroplasty: an updated meta-analysis of randomized controlled trials. J Orthop 16(4):294-302. https://doi.org/10.1016/j.jor.2019.03.003

55. Tan TL, Foltz C, Huang R, Chen AF, Higuera C, Siqueira M, Hansen EN, Sing DC, Parvizi J (2019) Potent anticoagulation does not reduce venous thromboembolism in high-risk patients. J Bone Joint Surg Am 101(7):589599. https://doi.org/10.2106/JBJS.18.00335

56. Matharu GS, Kunutsor SK, Judge A, Blom AW, Whitehouse MR (2020) Clinical effectiveness and safety of aspirin for venous thromboembolism prophylaxis after total hip and knee replacement: a systematic review and meta-analysis of randomized clinical trials. JAMA Intern Med 180(3):376-384. https://doi. org/10.1001/jamainternmed.2019.6108

57. Huang RC, Parvizi J, Hozack WJ, Chen AF, Austin MS (2016) Aspirin is as effective as and safer than warfarin for patients at higher risk of venous thromboembolism undergoing total joint arthroplasty. J Arthroplast 31(9): 83-86. https://doi.org/10.1016/j.arth.2016.02.074

58. Parvizi J, Huang R, Rezapoor M, Bagheri B, Maltenfort MG (2016) Individualized risk model for venous thromboembolism after total joint arthroplasty. J Arthroplast 31(9):180-186. https://doi.org/10.1016/j.arth.2016.02.077

59. National Institute for Health and Care Excellence (2018) Clinical Guidelines. In: Venous thromboembolism in over 16s: reducing the risk of hospitalacquired deep vein thrombosis or pulmonary embolism. National Institute for Health and Care Excellence, London

60. Mostafavi Tabatabaee R, Rasouli MR, Maltenfort MG, Parvizi J (2015) Costeffective prophylaxis against venous thromboembolism after total joint arthroplasty: warfarin versus aspirin. J Arthroplast 30(2):159-164. https://doi. org/10.1016/j.arth.2014.08.018

61. Dawoud DM, Wonderling D, Glen J, Lewis S, Griffin XL, Hunt BJ, Stansby G, Reed M, Rossiter N, Chahal JK, Sharpin C, Barry P (2018) Cost-utility analysis of venous thromboembolism prophylaxis strategies for people undergoing elective total hip and total knee replacement surgeries in the English National Health Service. Front Pharmacol 9:1370. https:/doi.org/10.3389/ fphar.2018.01370

62. Sobieraj DM, Lee S, Coleman Cl, Tongbram V, Chen W, Colby J, Kluger J, Makanji S, Ashaye AO, White CM (2012) Prolonged versus standard-duration venous thromboprophylaxis in major orthopedic surgery: a systematic review. Ann Intern Med 156(10):720-727. https://doi.org/10.7326/0003-481 9-156-10-201205150-00423

63. Nair V, Kumar R, Singh BK, Sharma A, Joshi GR, Pathak K (2013) Comparative study of extended versus short term thromboprophylaxis in patients undergoing elective total hip and knee arthroplasty in Indian population. Indian J Orthop 47(2):161-167. https://doi.org/10.41 03/0019-5413.108953

64. Parvizi J, Huang R, Raphael IJ, Maltenfort MG, Arnold WW, Rothman RH (2015) Timing of symptomatic pulmonary embolism with warfarin following arthroplasty. J Arthroplast 30(6):1050-1053. https://doi.org/10.1016/.jarth.2015.01.004

65. Pedersen AB, Andersen IT, Overgaard S, Fenstad AM, Lie SA, Gjertsen JE, Furnes $O$ (2019) Optimal duration of anticoagulant thromboprophylaxis in total hip arthroplasty: new evidence in 55,540 patients with osteoarthritis from the Nordic Arthroplasty Register Association (NARA) group. Acta Orthop 90(4):298-305. https://doi.org/10.1080/17453674.2019.1611215

66. Pedersen $A B$, Sorensen $H T$, Mehnert $F$, Johnsen SP, Overgaard S (2015) Effectiveness and safety of different duration of thromboprophylaxis in 16,865 hip replacement patients - a real-word, prospective observational study. Thromb Res 135(2):322-328. https://doi.org/10.1016/j.thromres.2014.11.029

67. Jiang Y, Du H, Liu J, Zhou Y (2014) Aspirin combined with mechanical measures to prevent venous thromboembolism after total knee arthroplasty: a randomized controlled trial. Chin Med J (Engl) 127:2201-2205

68. Zou Y, Tian S, Wang Y, Sun K (2014) Administering aspirin, rivaroxaban and low-molecular-weight heparin to prevent deep venous thrombosis after total knee arthroplasty. Blood Coagul Fibrinolysis 25(7):660-664. https://doi. org/10.1097/MBC.0000000000000121

69. Azboy I, Groff H, Goswami K, Vahedian M, Parvizi I (2020) Low-dose aspirin is adequate for venous thromboembolism prevention following total joint arthroplasty: a systematic review. J Arthroplast 35(3):886-892. https://doi. org/10.1016/j.arth.2019.09.043

70. Faour M, Piuzzi NS, Brigati DP, Klika AK, Mont MA, Barsoum WK, Higuera CA (2019) No difference between low- and regular-dose aspirin for venous thromboembolism prophylaxis after THA. Clin Orthop Relat Res 477(2):396402. https://doi.org/10.1097/CORR.0000000000000613

71. Faour M, Piuzzi NS, Brigati DP, Klika AK, Mont MA, Barsoum WK, Higuera CA (2018) Low-dose aspirin is safe and effective for venous thromboembolism prophylaxis following total knee arthroplasty. J Arthroplast 33(7):S131-S1s5. https://doi.org/10.1016/j.arth.2018.03.001

72. Panvizi J, Huang R, Restrepo C, Chen AF, Austin MS, Hozack WJ, Lonner JH (2017) Low-dose aspirin is effective chemoprophylaxis against clinically important venous thromboembolism following total joint arthroplasty: a preliminary analysis. J Bone Joint Surg Am 99(2):91-98. https://doi.org/10.2106/JBJS.16.00147

73. Fuji T, Ochi T, Niwa S, Fujita S (2008) Prevention of postoperative venous thromboembolism in Japanese patients undergoing total hip or knee arthroplasty: two randomized, double-blind, placebo-controlled studies with three dosage regimens of enoxaparin. J Orthop Sci 13(5):442-451. https:// doi.org/10.1007/s00776-008-1264-0

74. Mihara M, Tamaki Y, Nakura N, Takayanagi S, Saito A, Ochiai S, Hirakawa K (2020) Clinical efficacy of risk-stratified prophylaxis with low-dose aspirin for the management of symptomatic venous thromboembolism after total hip arthroplasty. J Orthop Sci 25(1):156-160. https://doi.org/10.1016/j.jos.2019.02. 009

\section{Publisher's Note}

Springer Nature remains neutral with regard to jurisdictional claims in published maps and institutional affiliations.
Ready to submit your research? Choose BMC and benefit from:

- fast, convenient online submission

- thorough peer review by experienced researchers in your field

- rapid publication on acceptance

- support for research data, including large and complex data types

- gold Open Access which fosters wider collaboration and increased citations

- maximum visibility for your research: over $100 \mathrm{M}$ website views per year

At BMC, research is always in progress.

Learn more biomedcentral.com/submissions 